\title{
Prevention of COPD exacerbation by lysozyme: a double-blind, randomized, placebo-controlled study
}

\author{
Yoshinosuke Fukuchi' \\ Koichiro Tatsumi ${ }^{2}$ \\ Hiromasa Inoue 3 \\ Yukinori Sakata ${ }^{4}$ \\ Kai Shibata ${ }^{4}$ \\ Hideaki Miyagishi ${ }^{4}$ \\ Yasuhiro Marukawa ${ }^{4}$ \\ Masakazu Ichinose ${ }^{5}$ \\ 'Department of Respiratory \\ Medicine, Graduate School of \\ Medicine, Juntendo University, Tokyo, \\ ${ }^{2}$ Department of Respirology, Graduate \\ School of Medicine, Chiba University, \\ Chiba, ${ }^{3}$ Department of Pulmonary \\ Medicine, Graduate School of Medical \\ and Dental Sciences, Kagoshima \\ University, Kagoshima, ${ }^{4}$ Eisai Co., Ltd., \\ Tokyo, ${ }^{5}$ Department of Respiratory \\ Medicine, Tohoku University Graduate \\ School of Medicine, Sendai, Japan
}

Correspondence: Yoshinosuke Fukuchi CRD Laboratory, Department of Respiratory Medicine, Graduate School of Medicine, Juntendo University, Asakaze BIdg 9F, 2-9-8 Hongo,

Bunkyo-ku, Tokyo, Japan

Tel/fax +8I 3 38I2 8473

Email yfukuchi@tea.ocn.ne.jp
This article was published in the following Dove Press journal:

International Journal of COPD

21 April 2016

Number of times this article has been viewed

Background/aim: Lysozyme (mucopeptide $N$-acetyl-muramyl hydrolase) is widely used as a mucolytic and anti-inflammatory agent in Japan. We evaluated the effects of long-term lysozyme administration on COPD exacerbation.

Methods: In a 1-year, randomized, double-blind, placebo-controlled, parallel trial, patients with moderate-to-severe COPD and one or more episodes of COPD exacerbation in the previous year before enrollment were selected. Lysozyme $(270 \mathrm{mg})$ or placebo was administered orally for 52 weeks as an add-on to the standard therapies such as bronchodilators. COPD exacerbation, pulmonary function, and COPD assessment test scores were analyzed. An exacerbation was defined as worsening of more than one symptom of COPD (cough, sputum volume, purulent sputum, or breathlessness) leading to a change in medication. The primary endpoint was exacerbation rate.

Results: A total of 408 patients were randomly assigned to the lysozyme and placebo groups. The baseline characteristics were similar between the two groups. The exacerbation rate was not significantly different between the two groups (1.4 vs $1.2 ; P=0.292$, Poisson regression). However, a subgroup analysis showed that lysozyme might reduce exacerbation rate in patients with airway-dominant phenotype (1.2 vs 1.6). Moreover, the median time to first exacerbation was longer in patients with airway-dominant phenotype in the lysozyme group than that in the placebo group. The levels of improvement in forced expiratory volume in 1 second and COPD assessment test scores were not statistically different between the groups, but were always greater in the lysozyme group than in the placebo group over the 52 weeks of the study.

Conclusion: The effects of using lysozyme as an add-on to standard COPD therapy were not significantly different compared with placebo and were insufficient to prevent COPD exacerbation.

Keywords: lysozyme, COPD exacerbation, forced expiratory volume in 1 second, COPD assessment test

\section{Introduction}

COPD is a pulmonary disease that presents with chronic symptoms such as cough, sputum, and dyspnea and causes airflow obstruction. Since this disease is pathologically progressive, prevention of exacerbation is important for the treatment of stable-phase COPD. The causes of COPD exacerbation are worsening of airway inflammation and increased sputum production. ${ }^{1}$ Moreover, the decline over time in airway obstruction is higher in COPD patients with mucus hypersecretion than in those without it. ${ }^{2}$ Thus, control of sputum production may be assumed to contribute to both resolution of pathological conditions and prevention of exacerbation in patients with COPD. 
Lysozyme is a bacteriolytic enzyme that was discovered by Alexander Fleming, the pioneer of penicillin, and exists widely in nature, including in humans and birds. Lysozyme is an enzyme with lytic action that is found in human secretions such as that produced by the eye, breast milk, and respiratory tract secretions. It is present in high levels in the respiratory epithelium and neutrophil granules and has antibacterial activity against gram-negative and gram-positive pathogens. ${ }^{3,4}$ Lysozyme has been shown to inhibit chemotaxis of the activated leukocytes. Other antiinflammatory functions of lysozyme include inhibition of mitogen-induced lymphoblastogenesis and autologous mixed lymphocyte reaction. ${ }^{5}$

In Japan, an egg white lysozyme preparation has long been used in routine clinical practice as an oral mucolytic agent for bronchitis with expectoration. Ciliary hypermotility and the promotion of foreign material excretion induced by lysozyme in the airway have been reported in animal models. ${ }^{6,7}$ The results of a clinical pharmacological trial suggested that sputum excretion is promoted in patients with chronic respiratory diseases by changing the properties of the sputum. ${ }^{8}$ Furthermore, a recent double-blind, placebo-controlled, crossover study on patients with COPD demonstrated significantly greater improvements in forced expiratory volume in 1 second $\left(\mathrm{FEV}_{1}\right)$, impulse-oscillationsystem-assessed peripheral pulmonary function, and COPD assessment test (CAT) scores in those receiving a combination of lysozyme with regular treatment than in those receiving regular treatment alone. ${ }^{9}$

Carbocysteine and $\mathrm{N}$-acetylcysteine, which facilitate expectoration, have been shown to have preventive effects on COPD exacerbation. ${ }^{10,11}$ Thus, the current study was conducted in patients with COPD receiving bronchodilators and other standard therapeutic agents under actual clinical conditions to investigate whether the add-on treatment with lysozyme, a mucolytic agent, would prevent COPD exacerbation.

\section{Materials and methods Study design}

This was a multicenter, randomized, double-blind, placebo-controlled study conducted from August 2012 to January 2015 (ClinicalTrials.gov number, NCT01645800). The protocol was evaluated in terms of ethics, safety, and scientific validity and approved by the Institutional Review Board at each participating institution.

During the screening period (day 0-28), patients who had provided written consent were assessed by investigators at each medical institution for eligibility for the current study, and baseline characteristics such as time of initial COPD diagnosis, clinical phenotype, the presence or absence of concurrent bronchial asthma, and smoking history were recorded. The investigators were experts with a lot of experience in the diagnosis and treatment of COPD. In daily clinical practice in Japan, a definitive diagnosis of COPD is made using computed tomography (CT) in most cases, in addition to spirometry. The investigators classified COPD as airwayor emphysema-dominant phenotype on the basis of clinical symptoms (eg, cough and sputum) as well as findings on CT images of the patients (if available).

After the screening period, the patients were randomly assigned to either lysozyme or placebo treatment in a ratio of 1:1. Treatment with the study drugs was administered for 52 weeks after randomization, and lysozyme $(90 \mathrm{mg})$ or placebo was administered orally three times a day, before each meal.

Any bronchodilators and inhaled corticosteroids that the patients had been receiving before treatment with the study drugs were considered standard therapy and were maintained until the end of the treatment period, without changing the dosage of prescribed medicines.

The use of expectorant was prohibited until the end of the treatment period. Moreover, systemic corticosteroids, antibacterial agents, antitussive agents, and cold remedies, as well as bronchodilators and inhaled corticosteroids that were not included in the standard therapy, were not allowed except in the case of temporary use at the time of COPD exacerbation.

\section{Patients}

Patients were enrolled in this trial at 47 Japanese medical institutions. Those meeting the following conditions were eligible: age between $\geq 20$ years and $<85$ years; smoking history (+); post-bronchodilator ratio of $\mathrm{FEV}_{1}$ to forced vital capacity (FVC) of $<70 \%$ and $\mathrm{FEV}_{1}$ of $<80 \%$ predicted in the screening period; history of at least one episode of exacerbation within the previous 1 year; and diagnosis of COPD by a physician. Patients were excluded if they met any of the following criteria: history of COPD exacerbation within the 7 days before the start of oral administration of the study drugs; history of lung transplantation, pneumonectomy, or lung volume reduction surgery; egg allergy; domiciliary oxygen therapy; pneumonia or pulmonary tuberculosis; severe cardiovascular disorders; severe kidney disorders; severe hepatic disorders; severe hematological disorders; cancer; and alcohol or drug dependence. 


\section{Endpoints}

The primary endpoint was prevention of COPD exacerbation (as assessed by exacerbation rate and time to first exacerbation). An exacerbation was defined as a worsening of more than one symptom of COPD (cough, sputum volume, purulent sputum, or breathlessness) for $>1$ day leading to a change in medication. For 52 weeks, patients were asked to record the "presence of symptoms" in a symptom diary only when they felt worsening of their symptoms compared with their everyday symptoms before study enrollment. Any additional drug used for the treatment of COPD exacerbation was also recorded in the symptom diary. At 4-week interval follow-up visits, the investigators determined the presence or absence of COPD exacerbation on the basis of the records in the symptom diaries and the results of interviews with the patients.

The secondary endpoints were respiratory function assessed by spirometry and health status assessed by CAT. ${ }^{12}$ During spirometry, $\mathrm{FEV}_{1}, \% \mathrm{FEV}_{1}$, and $\mathrm{FVC}$ were measured after inhalation of a bronchodilator at the follow-up visits on weeks $12,24,36$, and 52 . To avoid variations in measurements, the follow-up visits for spirometry measurements were scheduled as close to the same time as each other as possible. At each follow-up visit, the same spirometer was used as far as possible. The spirometers used were calibrated before each measurement. For the CAT, the patients filled in and submitted CAT forms to the investigators at the 4-week interval follow-up visits.

\section{Statistical analysis}

The mean rates of COPD exacerbation in the lysozyme group and the placebo group were assumed to follow Poisson distributions of 0.92 and 1.24 , respectively; this was based on the absence of any previous clinical reports on lysozyme and COPD exacerbation, or the expectorant test result. The power of this test was calculated as $80 \%$ with a two-sided significance level of 5\% and 180 cases in each group. Assuming a dropout rate of $\sim 10 \%$, the final number of cases was set as 200 in each group (400 cases in total).

The rate of COPD exacerbation was compared between the lysozyme group and the placebo group by Poisson regression analysis, with the COPD exacerbation rate in the past 52 weeks $(1,2$, or $\geq 3)$ and treatment group as covariates and concurrent bronchial asthma as a factor. Exacerbation rates of 5 or more episodes in 52 weeks were recorded as five episodes occurring in that period.

For time to first exacerbation, the patients were stratified by the rate of COPD exacerbation in the previous 52 weeks $(1,2$, or $\geq 3)$ and the presence or absence of concomitant bronchial asthma and then compared between the treatment groups with a stratified log-rank test. Hazard ratios were calculated with stratified Cox regression models.

For spirometry $\left(\mathrm{FEV}_{1}, \% \mathrm{FEV}_{1}\right.$, and $\left.\mathrm{FVC}\right)$, measurements were taken at each assessment point in each treatment group, and the changes from pretreatment levels (measurement at each assessment point - pretreatment level) were calculated. The changes were then compared between the placebo and lysozyme groups with $t$-tests.

For the CAT, scores for each question item and the total scores for all items were determined at each assessment point in each treatment group, and the changes from pretreatment scores (score at each assessment point - pretreatment score) were calculated. The changes were then compared between the placebo and lysozyme groups with $t$-tests.

All analyses were performed using SAS version 9.2 or greater (SAS Institute Inc., Cary, NC, USA).

\section{Results}

\section{Patient characteristics}

Although 408 patients were randomly assigned to the treatment groups after providing written consent, 406 patients (202 in the lysozyme group and 204 in the placebo group) received the study drugs, since two patients were withdrawn from the study before the start of oral administration. While 66 patients (31 in the lysozyme group and 35 in the placebo group) were withdrawn from the current study before its completion, 340 patients (171 in the lysozyme group and 169 in the placebo group) completed the 52-week treatment.

The baseline characteristics of the 406 patients are shown in Table 1. During the previous 52 weeks before the study, the mean rate of COPD exacerbation and the number of episodes of COPD exacerbation resulting in hospitalization or a visit to the emergency outpatient department were greater in the lysozyme group than in the placebo group.

\section{Efficacy}

\section{Exacerbation rate}

Exacerbation rate (mean \pm standard deviation) was $1.4 \pm 1.5$ in the lysozyme group and $1.2 \pm 1.4$ in the placebo group; these rates were not significantly different $(P=0.292$; Table 2$)$. There were 13 emergency visits and 40 hospitalizations related to COPD exacerbation. The COPD exacerbation rate was $0.1 \pm 0.4$ in the lysozyme group and $0.1 \pm 0.3$ in the placebo group $(P=0.556)$. Numbers of exacerbations per patient per year were not significantly different between the two groups (1.8 \pm 2.9 in the lysozyme group and $1.6 \pm 2.5$ in the placebo group; $P=0.305)$. 
Table I Baseline patient characteristics

\begin{tabular}{|c|c|c|}
\hline \multirow[t]{2}{*}{ Characteristics } & \multirow{2}{*}{$\frac{\text { LYS } 270 \mathrm{mg}}{(\mathrm{n}=202)}$} & \multirow{2}{*}{$\frac{\text { Placebo }}{(n=204)}$} \\
\hline & & \\
\hline \multicolumn{3}{|l|}{ Sex, $n$} \\
\hline Male & 183 & 186 \\
\hline Female & 19 & 18 \\
\hline Age (years), mean $\pm S D$ & $68.8 \pm 9.3$ & $70.3 \pm 7.2$ \\
\hline \multicolumn{3}{|l|}{ Duration of COPD } \\
\hline Years, mean $\pm S D$ & $4.6 \pm 4.2$ & $5.0 \pm 4.6$ \\
\hline$<2$ years, $\mathrm{n}$ & 40 & 35 \\
\hline$\geq 2$ years, $n$ & 162 & 169 \\
\hline \multicolumn{3}{|l|}{ Subtype of COPD, $\mathrm{n}$} \\
\hline Airway dominant & 49 & 43 \\
\hline Emphysema & 153 & 161 \\
\hline \multicolumn{3}{|l|}{ Smoking status, $\mathrm{n}$} \\
\hline Current & 62 & 61 \\
\hline Ex & 140 & 143 \\
\hline \multicolumn{3}{|l|}{ Gold stage, $n$} \\
\hline II & 119 & 133 \\
\hline III & 73 & 60 \\
\hline IV & 10 & 11 \\
\hline Complicated bronchial asthma, n (\%) & $90(44.6)$ & $95(53.4)$ \\
\hline Exacerbations in past 52 weeks, mean $\pm S D$ & $1.8 \pm 1.0$ & $1.6 \pm 1.0$ \\
\hline One, n (\%) & $107(53.2)$ & $122(59.8)$ \\
\hline Two or more, $\mathrm{n}(\%)$ & $94(46.8)$ & $82(40.2)$ \\
\hline $\begin{array}{l}\text { Hospitalization or emergency admission } \\
\text { in past } 52 \text { weeks, } n(\%)\end{array}$ & $29(14.4)$ & $21(10.3)$ \\
\hline \multicolumn{3}{|l|}{ Pulmonary function, mean \pm SD } \\
\hline $\mathrm{FEV}_{1}(\mathrm{~L})$ & $1.42 \pm 0.5$ & $\mathrm{I} .4 \mathrm{I} \pm 0.5$ \\
\hline Predicted FEV $(\%)$ & $54.39 \pm 15.8$ & $54.76 \pm 15.0$ \\
\hline $\mathrm{FVC}(\mathrm{L})$ & $2.85 \pm 0.8$ & $2.81 \pm 0.7$ \\
\hline $\begin{array}{l}\text { COPD assessment test (total score), } \\
\text { mean } \pm \text { SD }\end{array}$ & $12.9 \pm 6.6$ & $13.4 \pm 7.3$ \\
\hline \multicolumn{3}{|l|}{ GOLD classification, $\mathrm{n}(\%)$} \\
\hline$A$ & $18(9.0)$ & $23(11.3)$ \\
\hline B & $46(22.9)$ & $52(25.5)$ \\
\hline $\mathrm{C}$ & $43(21.4)$ & $44(21.6)$ \\
\hline $\mathrm{D}$ & $94(46.8)$ & $85(41.7)$ \\
\hline \multicolumn{3}{|l|}{ Standard treatment (multiple count), n (\%) } \\
\hline LABA & $89(44.1)$ & $83(40.7)$ \\
\hline LAMA & $136(67.3)$ & $15 \mathrm{I}(74.0)$ \\
\hline ICS/LABA & $118(58.4)$ & $123(60.3)$ \\
\hline ICS & $17(8.4)$ & $15(7.4)$ \\
\hline Xanthine & $58(28.7)$ & $58(28.4)$ \\
\hline
\end{tabular}

Abbreviations: GOLD, Global Initiative for Chronic Obstructive Lung Disease; $\mathrm{FEV}_{1}$, forced expiratory volume in I second; FVC, forced vital capacity; ICS, inhaled corticosteroid; LABA, long-acting beta-agonist; LAMA, long-acting muscarinic antagonist; LYS, lysozyme; SD, standard deviation.

Subgroup analyses showed that, in comparison to the placebo group, the mean rate of COPD exacerbation was lower in the following lysozyme subgroups: patients with a COPD duration of $<2$ years from the time of diagnosis ( 0.7 in the lysozyme group and 1.0 in the placebo group; $P=0.142$ ), patients with airway-dominant phenotype of COPD (1.2 and 1.6, respectively; $P=0.128$ ), and former or current smokers (1.0 and 1.2, respectively; $P=0.198$; Table 2).
Table 2 Exacerbation frequency

\begin{tabular}{llll}
\hline Group & LYS & Placebo & $P$-value \\
\hline All patients $(n=405)$ & 1.4 & 1.2 & 0.292 \\
$\begin{array}{l}\text { Duration of COPD }<2 \text { years } \\
(n=75)\end{array}$ & 0.7 & 1.0 & 0.142 \\
Airway dominant $(n=91)$ & 1.2 & 1.6 & 0.128 \\
Current smoker $(n=122)$ & 1.0 & 1.2 & 0.198 \\
\hline
\end{tabular}

Note: A Poisson regression analysis was performed, $P<0.05$. Abbreviation: LYS, lysozyme.

The COPD exacerbation rate was not significantly different between the group that used standard therapeutic agents including inhaled corticosteroids and the group that used standard therapeutic agents not including inhaled corticosteroids (data not shown).

\section{Time to first exacerbation}

The time to first exacerbation (median) was 179.0 days in the lysozyme group and 210.0 days in the placebo group; these results were not significantly different (hazard ratio, 1.06; $P=0.626$; Figure 1A).

According to subgroup analyses, the time to first exacerbation was $>365$ days in the lysozyme group and 219 days in the placebo group in patients with a COPD duration of $<2$ years from the time of diagnosis (hazard ratio, 0.66); 229.5 days and 161.0 days, respectively, in patients with airway-dominant phenotype (hazard ratio, 0.62); and 247.0 days and 238.0 days, respectively, in current smokers (hazard ratio, 0.75 ). Although administration of lysozyme prolonged the time to first exacerbation compared with placebo, no significant difference was observed between the lysozyme and placebo groups in any of the subgroups (Figure 1B and C).

\section{Spirometry}

In the lysozyme group, $\mathrm{FEV}_{1}$ improved over the 52 weeks; in addition, changes in $\mathrm{FEV}_{1}$ from baseline values ( \pm standard error [SE]) were always greater in the lysozyme group than in the placebo group, although this difference was not statistically significant. The changes at week 52 were $0.00 \pm 0.02 \mathrm{~L}$ in the lysozyme group and $-0.02 \pm 0.02 \mathrm{~L}$ in the placebo group ( $P=0.653$; Figure 2 ). In the lysozyme group, the improvement in $\mathrm{FEV}_{1}$ from baseline values (change $\pm \mathrm{SE}$ ) was greatest at week $12(0.04 \pm 0.02 \mathrm{~L}, P=0.173)$.

\section{COPD assessment test}

While the total CAT scores continued to improve from baseline to week 52 in both groups, the changes in the scores were greater in the lysozyme group than in the placebo group at all assessment points (Figure 3A). Moreover, the proportions of patients with total CAT scores that had improved by 1 point or more were higher in the lysozyme group than in the placebo 


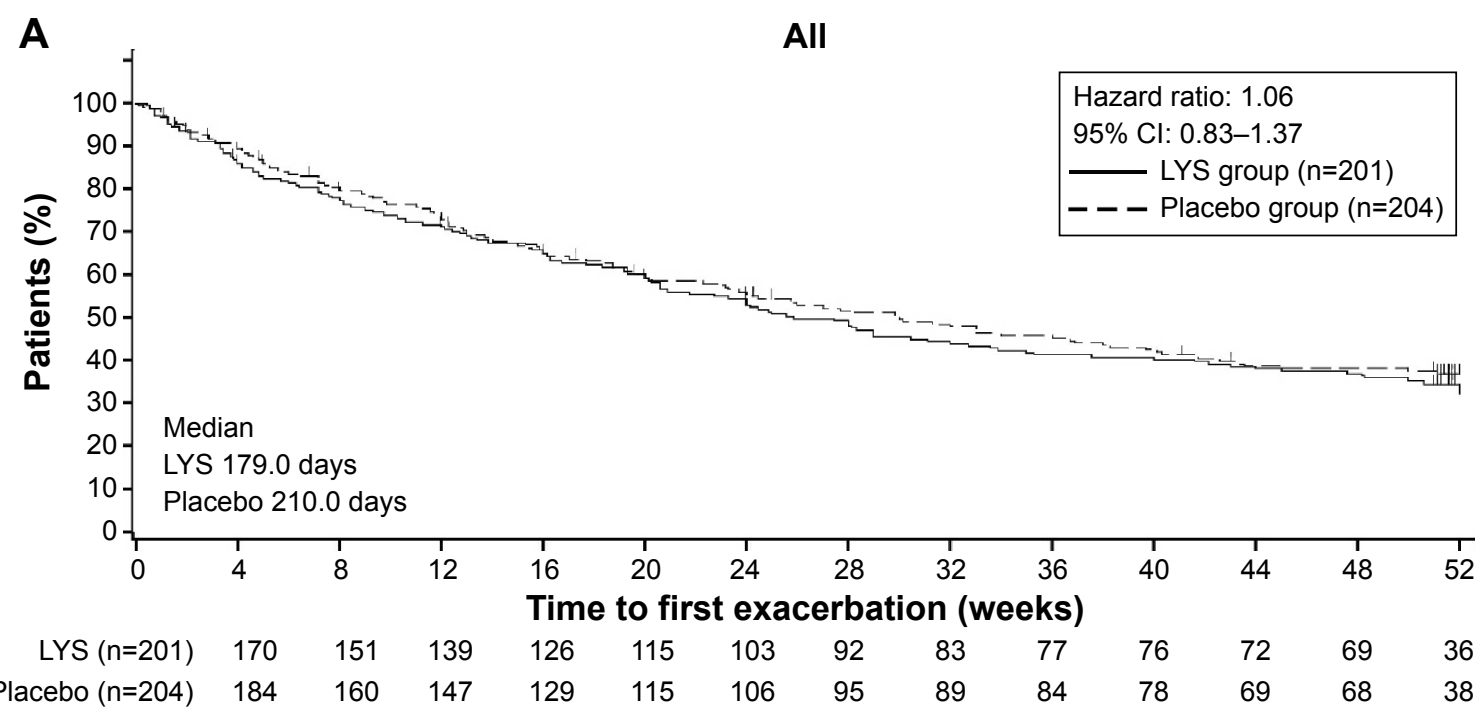

B

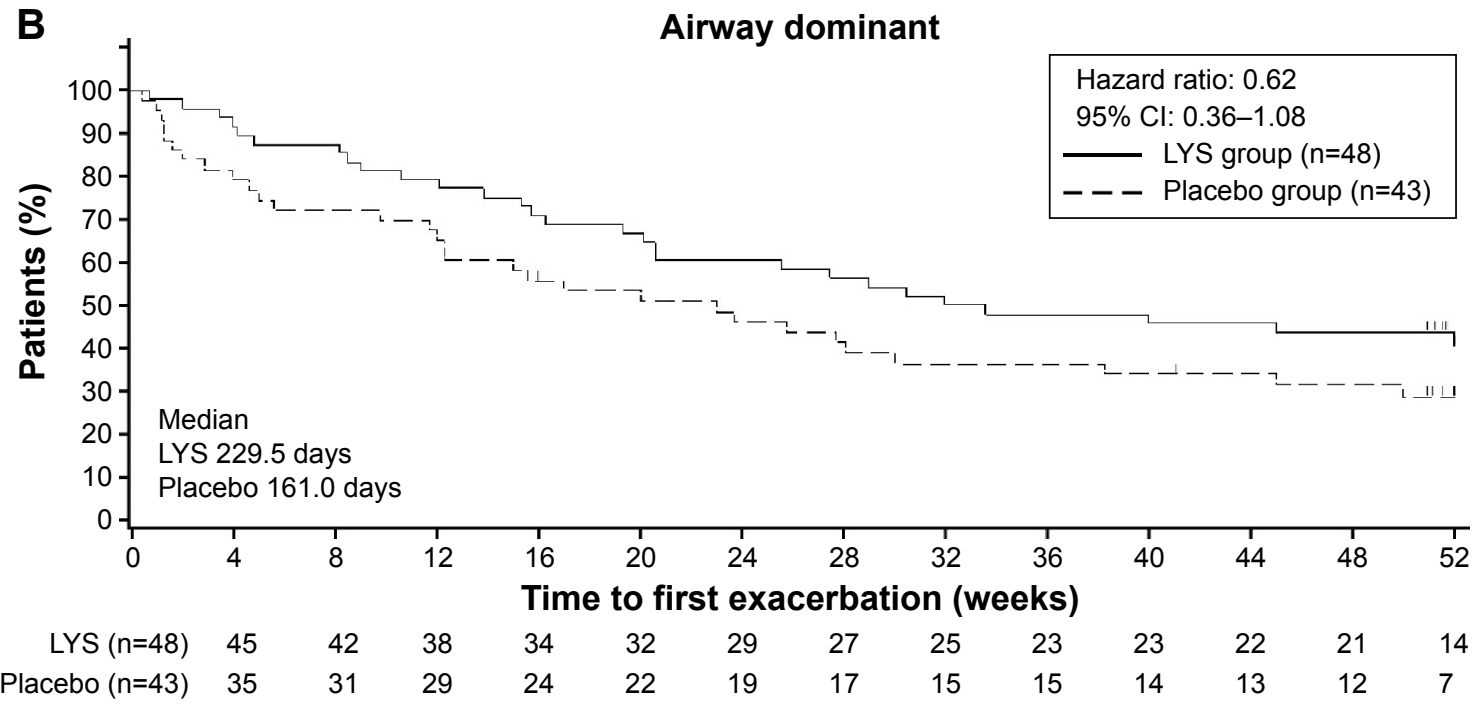

\section{Duration of COPD: <2 years}

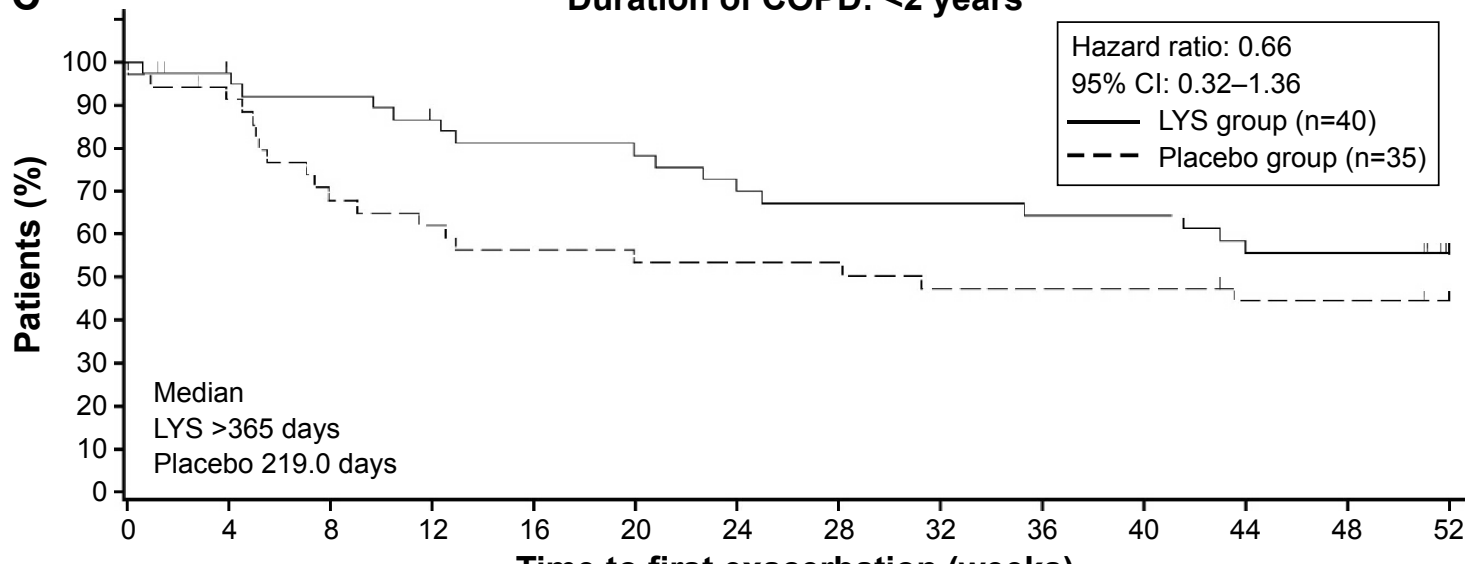

Time to first exacerbation (weeks)

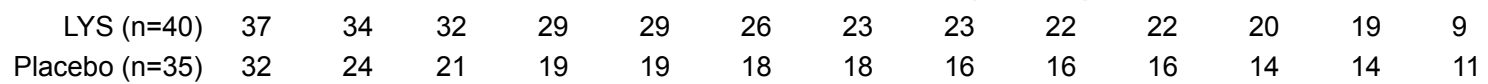

Figure I Kaplan-Meier plots showing the time to first exacerbation in (A) all patients, $(\mathbf{B})$ patients with airway-dominant phenotype, and $(\mathbf{C})$ patients with $<2$ years of disease duration.

Abbreviations: $\mathrm{Cl}$, confidence interval; LYS, lysozyme. 


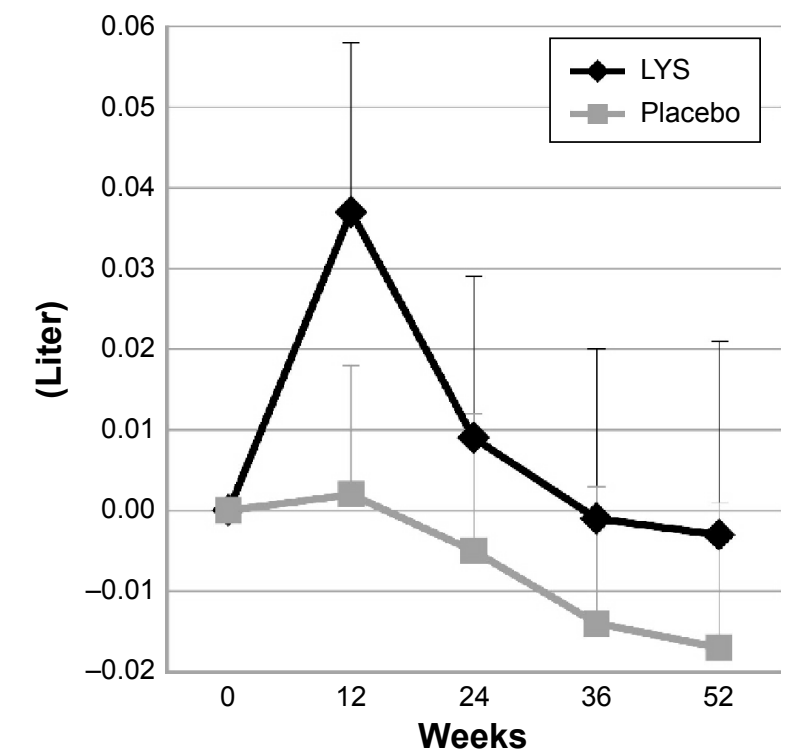

Figure 2 Changes in FEV, from the baseline (mean \pm standard error). Abbreviations: $\mathrm{FEV}_{1}$, forced expiratory volume in I second; LYS, lysozyme.

group at both weeks 12 and 24, and the difference between the groups was significant at week $24(P=0.027)$. Likewise, the proportions of patients with total CAT scores that had improved by 2 points or more were higher in the lysozyme group than in the placebo group at both weeks 12 and 24, and the difference between the groups was significant at week $12(P=0.033)$.

Temporal fluctuations in the changes in CAT scores for individual CAT question items from baseline scores were compared between the lysozyme and placebo groups. Of the eight CAT question items, the scores for both question 2 (I always feel my throat congested with sputum) and question 4 (I get severe breathlessness after climbing a slope or stairs) were significantly improved in the lysozyme group than in the placebo group $(P=0.006$ and $P=0.002$, respectively; Figure $3 \mathrm{~B}$ and $\mathrm{C}$ ).

\section{Safety}

Three patients (1.5\%) in the lysozyme group and five patients $(2.5 \%)$ in the placebo group had adverse events regarded by the investigators as being possibly related to the study drug. The major class of adverse drug reactions was gastrointestinal problems.

\section{Discussion}

To our knowledge, the current study is the first to investigate the preventive effects of lysozyme on COPD exacerbation
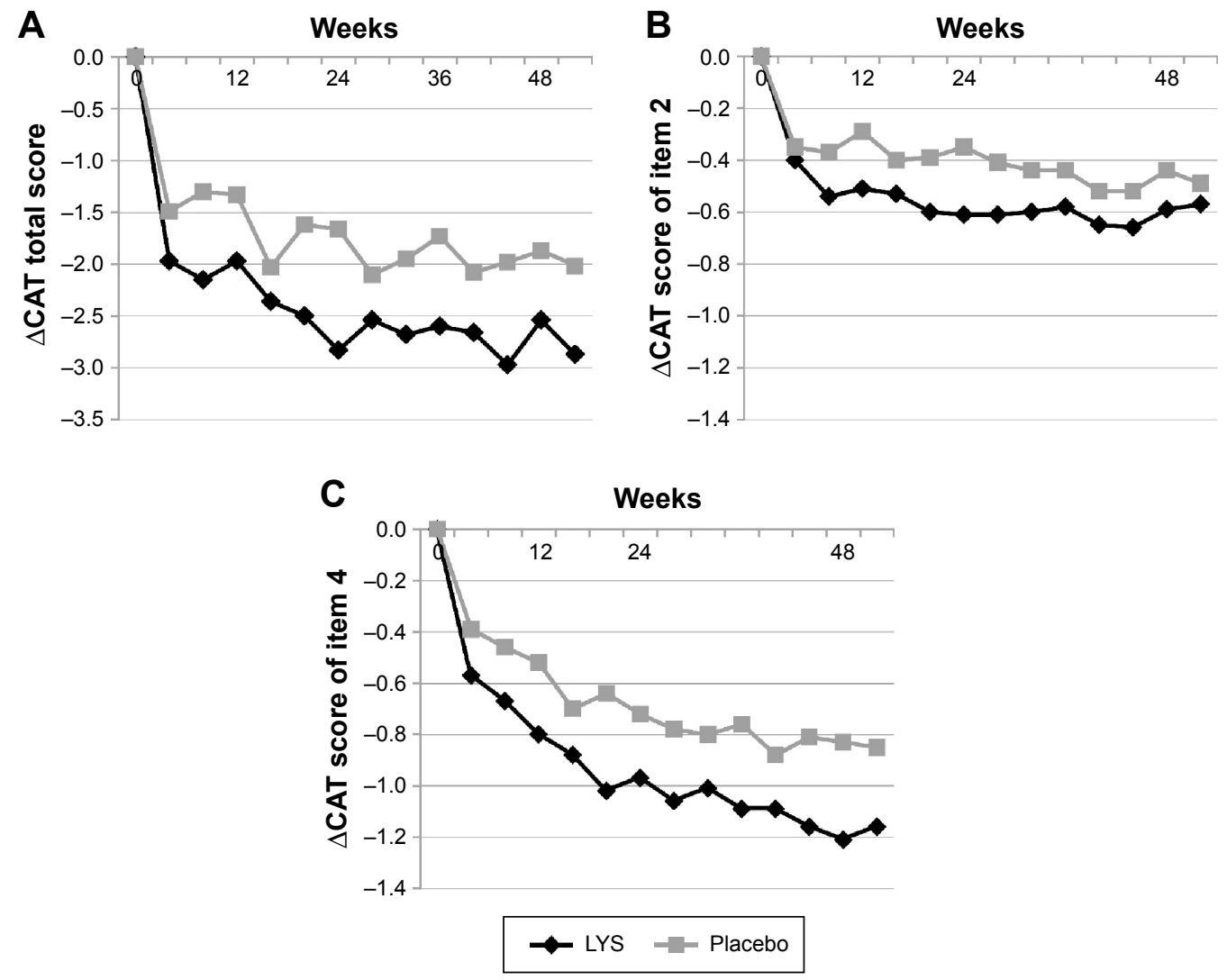

Figure 3 Mean changes in CAT scores from baseline.

Note: (A) Summary score, (B) item 2 (mucus volume), and (C) item 4 (breathlessness).

Abbreviations: CAT, COPD assessment test; LYS, lysozyme. 
in the world. In this study, we examined whether add-on treatment with $270 \mathrm{mg} / \mathrm{d}$ lysozyme to standard therapeutic agents for COPD had preventive effects on COPD exacerbation in patients with moderate-to-severe COPD. However, the primary endpoint was not fully met.

In recent years, COPD has been recognized as a complex condition composed of various clinical phenotypes, rather than a simple disorder of airflow obstruction, and personalized care has been emphasized. ${ }^{13}$ In the current study, common criteria for determining airway-or emphysemadominant phenotype were not set; instead, the individual investigators diagnosed the patients as having either phenotype of COPD. In the subgroup of patients with airwaydominant phenotype, the results suggested that lysozyme might prevent COPD exacerbation. If the therapeutic effects of lysozyme are investigated in a patient population further subdivided according to disease types of COPD in the future, the preventive effects of lysozyme on COPD exacerbation might well be validated.

COPD exacerbation was defined in the study of Carbocysteine on acute exacerbation of $\mathrm{COPD}^{10}$ as the condition when COPD-related symptoms occur on the basis of the definition developed by Anthonisen et al. ${ }^{14}$ However, in the current study, COPD exacerbation was defined as the condition when worsening of COPD-related symptoms resulted in changes in medication. Thus, because the latter definition includes only more severe cases of exacerbation, the number of cases of exacerbation based on the definition used in the current study was likely to be smaller than that based on the former definition. The proportion of patients with Global Initiative for Chronic Obstructive Lung Disease (GOLD) stage II disease was higher at $62.1 \%$ in the current study, as compared with $48.7 \%$ in the PEACE study. Despite this, the number of exacerbations per patient per year in the PEACE study was 1.01 in the active drug group and 1.35 in the placebo group, whereas the rate in the current study was 1.8 in the lysozyme group and 1.6 in the placebo group. Thus, the current study is assumed to include more patients with a higher exacerbation risk and more severe exacerbation symptoms as compared with the PEACE study. In such a patient population, the add-on effects of lysozyme might have been underestimated.

In contrast, spirometry and CAT, which were the secondary endpoints of the current study, may have suggested the presence of add-on effects of lysozyme. Since inflammatory responses of the airway are associated with the pathology of COPD, respiratory function can be assumed to have been improved by alleviation of inflammation by the antiinflammatory effects of lysozyme, the detailed mechanism of which is not known. ${ }^{15,16}$ Moreover, because of decreased ciliary motility of the respiratory mucosa in patients with COPD, ${ }^{17}$ $\mathrm{FEV}_{1}$ is lower in those with increased secretion from the airway because of the presence of inflammatory cells. ${ }^{18}$ It is also plausible that improved expectoration alone via the enhancing effects of lysozyme on ciliary transport ${ }^{3,4}$ might have contributed to the reduction in decrease in $\mathrm{FEV}_{1}$. The annual decrease in $\mathrm{FEV}_{1}$ in Japanese subjects has been reported to be -0.032 L. ${ }^{19}$ Moreover, a study conducted on carbocysteine in Japan showed that changes in $\mathrm{FEV}_{1}$ at 6 months after treatment were $-0.02 \mathrm{~L}$ in the carbocysteine group and $-0.03 \mathrm{~L}$ in the placebo group. In comparison to these results, the current study showed that changes in $\mathrm{FEV}_{1}$ at 24 weeks after beginning the treatment were $0.009 \mathrm{~L}$ in the lysozyme group and $-0.005 \mathrm{~L}$ in the placebo group. Thus, lysozyme appears to be comparable to carbocysteine in its preventive effect on the temporal decrease in $\mathrm{FEV}_{1}$ in patients with COPD.

In recent years, drug evaluation based on patient-centered outcomes has been emphasized. The American Thoracic Society and the European Respiratory Society also issued a joint recommendation to promote the use of patient-centered outcomes in clinical studies in the field of COPD. ${ }^{20}$ The CAT, which is used in Japan and many other countries in the world, is a patient-completed questionnaire for subjective symptoms and quality of life. It is one of the typical methods to evaluate drugs for COPD based on patient-centered outcomes. ${ }^{21}$ An improvement of two or more points in the CAT is considered the minimum clinically important difference. ${ }^{22}$ The proportion of patients who improved two or more points in this study in the lysozyme group was significantly higher than that in the placebo group only in week 12 of the 52 weeks. However, the number of improvement points was always greater in the lysozyme group than in the placebo group during the 52 weeks. Thus, the addition of lysozyme might be meaningful within the bronchodilator-based treatment scheme for COPD.

\section{Limitation}

This study had some limitations. It is known that orally administered lysozyme is absorbed in the intestinal tract, ${ }^{23}$ but the lysozyme concentration in the lungs and the lysozyme receptor distribution in the lungs have not been clarified. In addition, although the investigators are experts in COPD diagnosis, the classification of COPD as airway- or emphysema-dominant could have been judged differently by each investigator.

\section{Conclusion}

In conclusion, the current study showed that the effect of add-on administration of oral lysozyme to standard therapy 
was not significantly different to placebo and was insufficient for the prevention of COPD exacerbation. Further studies are needed to investigate whether $\mathrm{FEV}_{1}, \mathrm{CAT}$, and the add-on effects of lysozyme affect the time to first COPD exacerbation.

\section{Acknowledgments}

We would like to express our appreciation to the principal investigators and their staff at the 47 Japanese medical institutions who participated in the current study. This study was conducted with funds from Aska Pharmaceutical Co., Ltd., Nippon Shinyaku Co., Ltd., and Eisai Co., Ltd.

\section{Disclosure}

YF, MI, KT, and HI are consultants for Eisai Co., Ltd. Moreover, KS, HM, YM, and YS are employees of Eisai Co., Ltd. The authors report no other conflicts of interest in this work.

\section{References}

1. Burgel PR, Nesme-Meyer P, Chanez P, et al; Initiatives Bronchopneumopathie Chronique Obstructive Scientific Committee. Cough and sputum production are associated with frequent exacerbations and hospitalizations in COPD subjects. Chest. 2009;135(4):975-982.

2. Vestbo J, Prescott E, Lange P. Association of chronic mucus hypersecretion with $\mathrm{FEV}_{1}$ decline and chronic obstructive pulmonary disease morbidity. Copenhagen City Heart Study Group. Am J Respir Crit Care Med. 1996;153(5):1530-1535.

3. Dajani R, Zhang Y, Taft PJ, et al. Lysozyme secretion by submucosal glands protects the airway from bacterial infection. Am J Respir Cell Mol Biol. 2005;32(6):548-552.

4. Ellison RT 3rd, Giehl TJ. Killing of gram-negative bacteria by lactoferrin and lysozyme. J Clin Invest. 1991;88(4):1080-1091.

5. Gordon LI, Douglas SD, Kay NE, Yamada O, Osserman EF, Jacob HS. Modulation of neutrophil function by lysozyme. Potential negative feedback system of inflammation. J Clin Invest. 1979;64(1):226-232.

6. Shimizu K, Kondo M, Yonebayashi S, Sakuma Y, Taira Y. Effects of lysozyme chloride $\left(\right.$ Acdeam $\left.^{\circledR}\right)$ on foreign materials excretion by tracheal mucociliary transport. Jpn Pharmacol Ther. 1997;25(9):2347-2358.

7. Kayama S, Fukuchi Y, Harasawa M. An experimental study in the influence of age various drugs on ciliary beat frequency. Jpn Pharmacol Ther. 1985;13(2):701-705.

8. Tanaka K, Mikami M, Ishikawa T, et al. The study of lysozyme chloride on the relationship between the chances of symptoms and physiochemical properties of sputum in chronic pulmonary disease. Jpn J Med Pharm Sci. 2005;53(5):581-600.
9. Ohbayashi H, Setoguchi Y, Fukuchi Y, Shibata K, Sakata Y, Arai T. Pharmacological effects of lysozyme on COPD and bronchial asthma with sputum: a randomized, placebo-controlled, cross-over study. Pulm Pharmacol Ther. 2016;37:73-80.

10. Zheng JP, Kang J, Huang SG, et al. Effect of carbocisteine on acute exacerbation of chronic obstructive pulmonary disease (PEACE study): a randomised placebo-controlled study. Lancet. 2008;371(9629): 2013-2018

11. Zheng JP, Wen FQ, Bai CX, et al; PANTHEON Study Group. Twice daily $\mathrm{N}$-acetylcysteine $600 \mathrm{mg}$ for exacerbations of chronic obstructive pulmonary disease (PANTHEON): a randomised, double-blind placebo-controlled trial. Lancet Respir Med. 2014;2(3):187-194.

12. Tsuda T, Suematsu R, Kamohara K, et al. Development of the Japanese version of the COPD assessment test. Respir Investig. 2012;50(2): 34-39.

13. Agusti A. The path to personalised medicine in COPD. Thorax. 2014; 69(9):857-864.

14. Anthonisen NR, Manfreda J, Warren CP, Hershfield ES, Harding GK, Nelson NA. Antibiotic therapy in exacerbation of chronic obstructive pulmonary disease. Ann Intern Med. 1987;106(2):196-204.

15. Naito S, Tominaga H, Uno J, Dohiguchi Y, Hamaguchi K. The antiinflammatory effect of lysozyme hydrochloride on rabbits with different doses. Jpn Pharmacol Ther. 1986;14(6):4165-4169.

16. Fukawa K, Irino O, Watanabe A. Pharmacological effects of lysozyme and its reaction with serum proteins. Pharmacometrics. 1981;21(4): 587-593.

17. Piatti G, Ambrosetti U, Santus P, Allegra L. Effects of salmeterol on cilia and mucus in COPD and pneumonia patients. Pharmacol Res. 2005;51(2):165-168.

18. Hogg J, Chu F, Utokaparch S, et al. The nature of small-airway obstruction in chronic obstructive pulmonary disease. $N$ Engl J Med. 2004;350(26):2645-2653.

19. Nishimura M, Makita H, Nagai K, et al; Hokkaido COPD Cohort Study Investigators. Annual change in pulmonary function and clinical phenotype in chronic obstructive pulmonary disease. Am J Respir Crit Care Med. 2012;185(1):44-52.

20. Celli BR, Decramer M, Wedzicha JA, et al; ATS/ERS Task Force for COPD. An official American Thoracic Society/European Respiratory Society statement: research questions in chronic obstructive pulmonary disease. Am J Respir Crit Care Med. 2015;191(7):e4-e27.

21. Cazzola M, Hanania NA, MacNee W, Rüdell K, Hackford C, Tamimi N. A review of the most common patient-reported outcomes in COPD revisiting current knowledge and estimating future challenges. Int J Chron Obstruct Pulmon Dis. 2015;10:725-738.

22. Kon SS, Canavan JL, Jones SE, et al. Minimum clinically important difference for the COPD assessment test: a prospective analysis. Lancet Respir Med. 2014;2(3):195-203.

23. Hashida S, Ishikawa E, Nakamichi N, Sekino H. Concentration of egg white lysozyme in the serum of healthy subjects after oral administration. Clin Exp Pharmacol Physiol. 2002;29(1-2):79-83.
International Journal of COPD

\section{Publish your work in this journal}

The International Journal of COPD is an international, peer-reviewed journal of therapeutics and pharmacology focusing on concise rapid reporting of clinical studies and reviews in COPD. Special focus is given to the pathophysiological processes underlying the disease, intervention programs, patient focused education, and self management protocols.

\section{Dovepress}

This journal is indexed on PubMed Central, MedLine and CAS. The manuscript management system is completely online and includes a very quick and fair peer-review system, which is all easy to use. Visit $\mathrm{http}: / / \mathrm{www}$.dovepress.com/testimonials.php to read real quotes from published authors. 